\title{
A preliminary study of the dental implant therapy - initial osteogenesis of Human Mesenchymal Stem (HMS0014) cells on titanium discs with different surface modifications -
}

\author{
By
Yasutomo IWAI, Yoshifumi MATSUDA, Michiko NAKATSUKA, Yutaka MIKAMI, and Shunji KUMABE

Department of Oral Anatomy, Osaka Dental University 8-1, Kuzuhahanazono-cho, Hirakata-shi, Osaka 573-1121, Japan

- Received for Publication, December 19, 2011 -

\begin{abstract}
Key Words: Osteogenesis, HMS0014 cell, peptide-based hydrogel scaffold, GBR, in vitro
Summary: HMS0014 cells were GBR-engineered to proliferate and differentiate into mature osteoblast(Ob)-like cells, which initiated hard tissue matrix deposition in both monolayer and PuraMatrix 3-D cultures. Subsequently, the osteogenesis initiated with attachment/adhesion of HMS0014 cells on either Titanium (Ti) or Ti alloy discs modified with osteoconductive/ osteoinductive surface textures/substrates (e.g., Disc-AO, Disc-HA, Disc-SPI) was histologically assessed. The results obtained were as follows: 1) The HMS0014 cells actively proliferated and differentiated into mature Obs to initiate mineralisation of the ECM since day 1 in both monolayer and 3-D cultures; mineralization was prominently progressed between day 7 and day 14 of cultures. 2) The SEM of 60-minute(min)s specimens demonstrated a loose distribution of proliferating spherical-to-polygonal (10 to $40 \mu \mathrm{m}$ in diameter, avg.) cells with a bulging cell body sending out many minute filopodia and some lamellipodia to attach with the substrate in the concavities. 3) In the 180-min specimens, the cultured HMS0014 cells actively proliferated and spread into flat, large polygonal cells with prominent lamellipodia and dendritic filopodia $(30 \mu \mathrm{m} \times 90 \mu \mathrm{m}$ to $100 \mu \mathrm{m} \times 200 \mu \mathrm{m}$, approx.) to employ cell-to-substrate and intercellular attachments. 4) On the other hand, the present immunohistochemistry of the attached HMS0014 cells demonstrated the co-expression of F-actin (actin filaments of the cytoskeleton) and CD51 ( $\alpha \mathrm{V}$ integrin) in both the 60-min and 180-min specimens. We concluded that the present GBR method enhanced HMS0014 cells to initiate an osteogenesis process with a direct bone-to-substratum contact on Ti discs which were subject to different surface modifications.
\end{abstract}

\section{Introduction}

Several previous studies on the dental implantology have elucidated that surface modifications with different microtextures of the implant (IP) fixture not only increased direct bone-to-implant contact (BIC) compatibility, but also acquired primary stability for immediate load bearing $^{1-8)}$. Other studies have also demonstrated that regulation of vital reactions and consequent modeling and remodeling at the BIC interfaces might rapidly organize functional osseointegration to obtain a long-term stability of IPs for the occlusal rehabilitation therapy ${ }^{8-13)}$.

Previously, we have isolated and cultured mesenchyme cells (e.g., rat/human dental pulp cells, rat bone marrowderived stromal (BMS) cells, mouse KUSA/A (JCRB/ HSRRB; Osaka, Japan) cells), and elucidated that they were engineered to activate certain mechanisms to initiate hard tissue matrix deposition in both monolayer and 3-D cultures based on the guided bone regeneration (GBR) concept $^{14-18)}$. Subsequently, we extend the studies to investigate osseointegration, including contact and distant osteogenesis, by 3-D culture of KUSA/A1 cells with a Cellmatrix type I-A (Nitta Gelatin, Osaka, Japan) collagen scaffold on titanium (Ti) discs and implants, which have commercially available surface textures and modifications (i.e., anodic oxidation: AO, hydroxyapatite coating: HA, precision blasting: SPI). Hence, we observed that the GBR method engineered mouse BMS cells in a 3-D collagenous scaffold to initiate an osteogeneic process of new bone formation without intervening soft tissue in the peri-IP osteogenesis, and hypothesized that this peri-IP osteoid tissue might be employed to a biocompatible and biodegradable tissue, which could consequently be remolded and modeled for functional osseointegration ${ }^{19,20)}$. 
PuraMatrix (3-D Matrix Japan, Ltd., Tokyo, Japan) is a biocompatible self-assembling peptide-based 3-D hydrogel scaffold, which produces a 3-D nano-fibrillar and porous environment to assist in providing a template for cell distribution and extracellular matrix (ECM) accumulation and thereby enhances cell-biomaterial interactions to promote growth and differentiation of cultured calf chondrocytes and rat osteoblasts ${ }^{21-25)}$. In light of these study results, in the present study, we performed monolayer culture and 3-D culture (with PuraMatrix) of HMS0014 cells (Human osteoblastic cell line, BM-derived mesenchymal cells) on Ti and Ti-alloy discs (i.e., Disc-AO, Disc-HA, Disc-SPI), aimed to develop a GBR environment which is beneficial for osseointegration to ensure an optimal direct boneto-substrate contact on the discs having similar surface modifications of prevalent dental implants, therefore contributed to a preliminary study of the dental implant therapy.

\section{Materilas \& Methods}

Characterization of osteoblast-like behavior of cultured HMS0014 cells

Immature HMS0014 cells (Human osteoblastic cell line, BM-derived mesenchymal cells) in non-induction condition were distributed through Riken BioResource Center, Tsukuba, Japan). The immature osteogeneic linage cells were filtrated, centrifuged, cultured and incubated in POWEREDBY 10 (Med Shirotori, Tokyo, Japan) supplemented with 1\% antibiotic-antimycotic agent: 100 units/ $\mathrm{ml}$ penicillin $+100 \mu \mathrm{g} / \mathrm{ml}$ streptomycin (Nacalai Tesque, Kyoto, Japan) in cell culture $75 \mathrm{~cm}^{2}$ flasks (TPP, Switzerland) at $37^{\circ} \mathrm{C}$ in humid air with $5 \% \mathrm{CO}_{2}$.

The immature HMS0014 cells were monolayer and 3-D cultured with 1\% PuraMatrix in the POWEREDBY 10 (Med Shirotori) added with ascorbic acid (AA) (50 $\mu \mathrm{m} / \mathrm{ml}$; Sigma-Aldrich Japan, Tokyo, Japan) and $\beta$-Glycerophosphate ( $\beta$-GP) (10 mM; Sigma-Aldrich) in $10 \%$ fetal bovine serum (HyClone, Logan, UT, USA) for 14 days. Subsequently, proliferation rate and differentiation of the monolayer-cultured cells and 3D-cultured cells were assayed and compared with PicoGreen dsDNA Quantitation Kit (Invitrogen, CA, USA; excitation wave length: $485 \mathrm{~nm}$, emission wave length: $525 \mathrm{~nm}$ ), Alkaline Phosphatase Substrate Kit (Bio-Rad Labs., CA, USA), Calcium E-test-Wako (Wako, Osaka, Japan) and the Gla type Osteocalcin (Gla-OC) EIA Kit (TaKaRa Bio, Ohtsu, Japan). The intensity of visible light (wave length: 405) emitted by the specimens was summarized and scored using a Softmax Pro5 (Molecular Devices, CA, USA). ALPase(ALP) activity expression was corrected for the total protein content (Bradford method using bovine serum albumin as a standard) by the DNA amount, and was expressed as $\mu \mathrm{mol} / \mu \mathrm{g} \mathrm{DNA} / \mathrm{min}$. On the other hand, the histology of the monolayer-cultured specimens and 3-D cultured cell/PuraMatrix clusters specimens were examined with a phase contrast microscope (CKX41; Olympus, Tokyo, Japan).

Tissue engineering of HMS0014 cells on discs with different surface modifications

Plates $(\mathrm{d}=1 \mathrm{~cm}, \mathrm{~h}=1 \mathrm{~mm}$; disc type $)$ of primarily blasted $\alpha-\beta$ type titanium alloy (Ti-6Al-4V) those were secondarily processed with anodic oxidization (JAPAN MEDICAL MATERIALS (JMM) Physio Odontrum Implant (POI) system: JMM, Osaka, Japan; Disc-AO) or coated with hydroxyapatite $\left(\mathrm{HA}=\mathrm{Ca}_{10}\left(\mathrm{PO}_{4}\right)_{6}(\mathrm{OH})_{2}\right.$, $20 \mu \mathrm{m}$ approx., JMM; Disc-HA) and JIS type 4 (99\% Ti) plates $(\mathrm{d}=1 \mathrm{~cm}, \mathrm{~h}=1 \mathrm{~mm}$; disc type $)$ finely blasted with $\mathrm{Al}_{2} \mathrm{O}_{3}$ sands (THOMMEN medical/Morita, Osaka, Japan: SPI; Disc-SPI) were supplied for the present in vitro study.

The isolated immature HMS0014 cells were seeded and cultured with the discs (i.e., Disc-AO, Disc-HA, DiscSPI) in the POWEREGBY 10 and exposed to AA, $\beta$-GP and dexametazone $(100 \mathrm{nM})$ at $1.0 \times 10^{5}$ cells $/ \mathrm{ml}$ concentration in 12-well petridishes (IWAKI, Tokyo, Jpn), thereby the cells were induced to differentiate into mature osteogeneic cells in a humid $5 \% \mathrm{CO}_{2}$ at $37^{\circ} \mathrm{C}$.

\section{The histology of HMS0014 cells cultured on plates}

1. Scanning electron microscopy (SEM)

Some 60-min and 180-min specimens (i.e., monolayer cultures of HMS0014 cells on Disc-AO, Disc-HA and Disc-SPI) were prefixed with $1 \%$ glutaraldehyde (20 min) and postfixed with $1 \% \mathrm{OsO}_{4}\left(30 \mathrm{~min}, 4^{\circ} \mathrm{C}\right)$ and prepared (Pt-Pd coating; Eiko IB-3 ion coater; Eiko engineering, Mito, Japan) for the conventional SEM. Secondary electron image SEM of the specimens was examined and digitally photographed under a Hitachi S-4000 SEM (accelerating voltage: $10 \mathrm{kV}$; Hitachi, Tokyo, Japan).

2. Immunohistochemical histology of HMS0014 cells on different surface modifications:

The 60- and 180-min specimens were immerse-fixed with $4 \%$ formaldehyde $\left(12 \mathrm{hrs}, 4^{\circ} \mathrm{C}\right)$, washed with $0.1 \%$ BSA-PBS and blocked with 3\% goat serum. The specimens were immunohistochemically stained with primary Ab: anti-CD51 ( $\alpha \mathrm{V}$ integrin, 1:1,000 Conc.; Enzo Life Sci, PA, USA) and secondary Abs: Alexa Fluor 488 goat anti-rabbit IgG (1:500 in 0.1\% BSA-PBS; Molecular probes, OR, USA), and subsequently stained with Rhodamine phalloidin (1:1000 in 0.1\% BSA-PBS; Molecular probes) and incubated (room temp., $1 \mathrm{hr}$ ) following the conventional methods. Therefore, the expression of CD51 and F-actin were examined, for evaluation of cell attachment and extension of the differentiating HMS0014 cells distributed on the discs subject to different surface modifications, with a BIOREVO BZ-9000 fluorescence microscopy (KEYENCE, Osaka, Japan). 


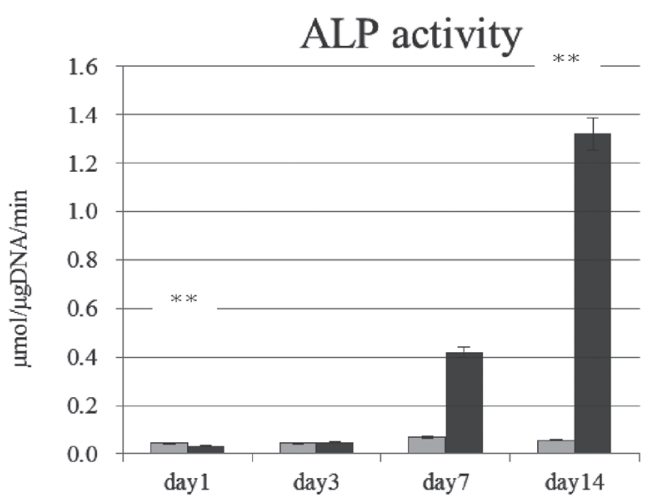

$\square$ control

Eexperiment

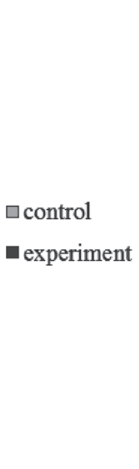

$\square$ control

- experiment

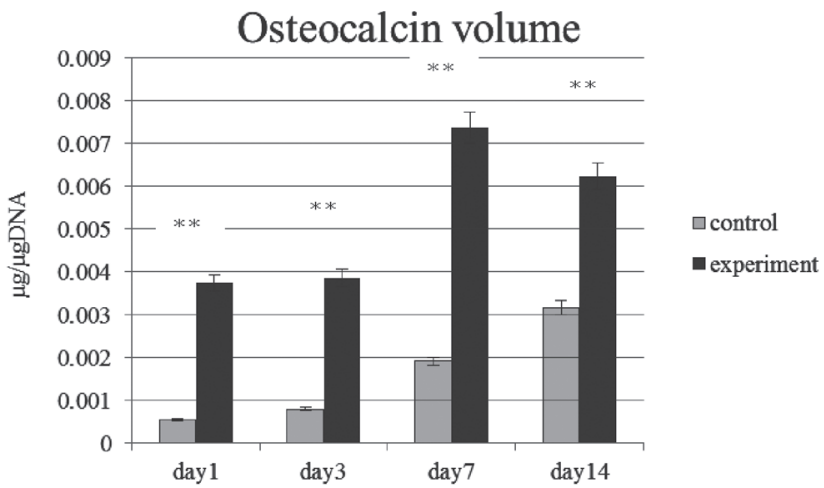

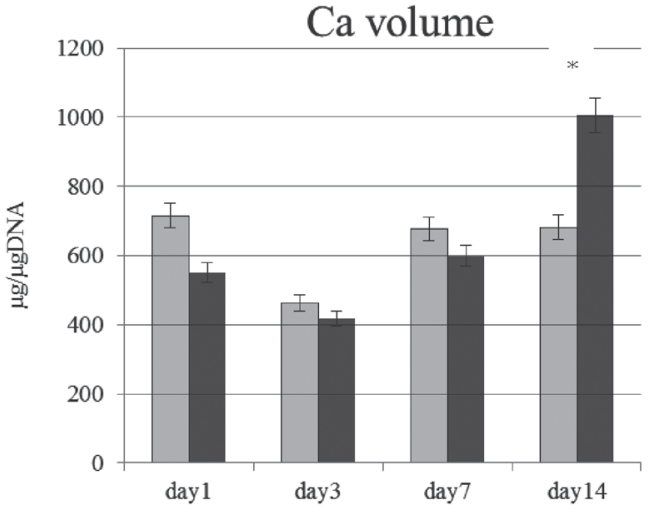

\section{Results}

Properties of the inducing-conditioned HMS0014 cells

The properties of proliferative HMS0014 cells were examined by analysis of ALP activity, Ca volume and osteocalcin (OC) volume (Fig. 1) in the accumulating ECM and by phase contrast microscopy of cells (Fig. 2) either in monolayer culture (Fig. 1A \& Fig. 2A) or in 3-D culture in PuraMatrix (Fig. 1B \& Fig. 2B) in vitro.

1. ALP activity, Ca volume and $\mathrm{OC}$ volume of monolayercultured HMS0014 cells (Fig. 1A)

1) By comparison of data obtained from the experimental group with that of the control group, we observed that the ALP activity increased with elapsed time, and the activity was markedly and significantly increased between day 7 and day 14 of the experiment $(* * p<0.01)$.

2) No significant differences in Ca volume was found between the experimental and control groups except on the day $14(* \mathrm{p}<0.05)$.

3) OC volume of the experimental group was significantly higher than that of the control group since the day 1 of culture $(* * \mathrm{p}<0.01)$. The graph indicated a tendency that the $\mathrm{OC}$ volume was markedly increased between day 3 and day 7 of experiment.
Fig. 1A. Monolayer culture of HMS0014 cells in vitro

1) ALP activity was markedly increased between day 7 and day 14 in the experimental group. A significant difference was in particular observed at 14 days experiment $(* * \mathrm{p}<0.01)$.

2) A significant increase of $\mathrm{Ca}$ volume was evident in the 14th day of experiment $\left({ }^{*} \mathrm{p}<0.05\right)$.

3) Significant differences of time-dependent increase of osteocalcin $(\mathrm{OC})$ volume were demonstrated $(* * \mathrm{p}<0.01)$.

The results indicated that the monolayer-cultured proliferating HMS0014 cells was actively induced to differentiate into Ob-like cells which abundantly secreted mineralizing ECM between day 3 and day 7 of culture; mineralization of the ECM prominently progressed between day 7 and day 14 (Fig. 1A).

2. ALP activity, Ca volume and OC volume of 3Dcultured HMS0014 cells in PuraMatrix scaffold (Fig. 1B) 1) The ALP activity of inducing-conditioned HMS0014 cells was found to be increased with elapsed time, and was significantly differed in the $14^{\text {th }}$ day of experiemnt $(* * p<0.01)$. However, the activity seemed to be a little lower than that had observed in the monolayer cultures at 14 days experiment (Fig. 1A).

2) An abrupt increase in $\mathrm{Ca}$ volume was found between day 7 and day 14 in the experimental group, thereby a significant difference was evident during the $14^{\text {th }}$ inducingconditioned day $(* \mathrm{p}<0.05)$. In addition, the Ca volume in 3 -D hydro-gel cultures was nearly $2 \times$ more increased than what was estimated in the monolayer cultures at 14 days experiments (Figs. 1A \& 1B).

3) No significant increase of OC volume was found in both the control and experimental groups, but an abrupt and significant increase of $\mathrm{OC}$ volume in the experimental 

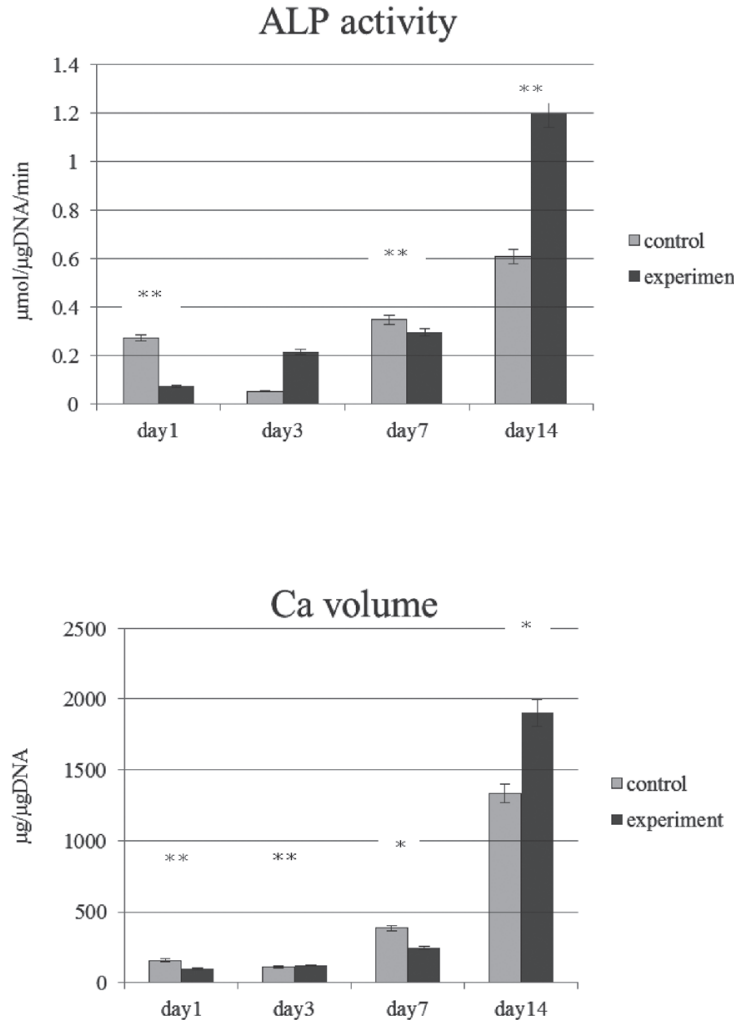

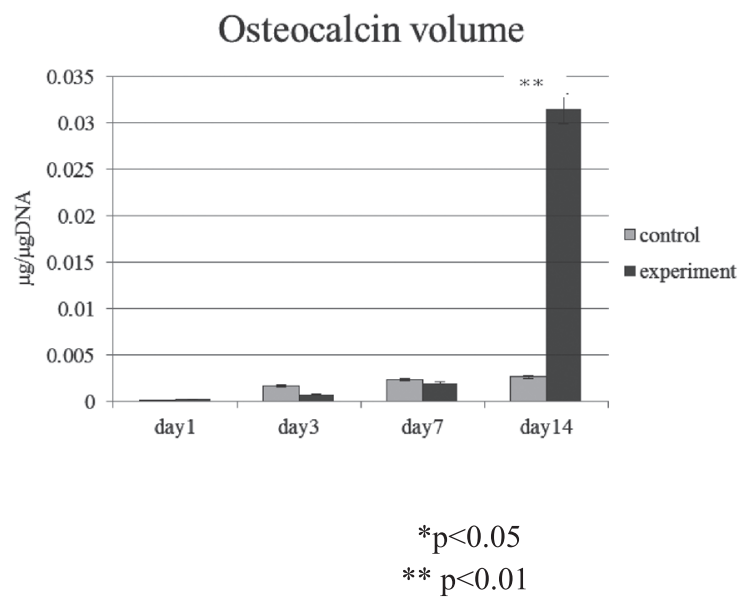

Fig. 1B. 3-D culture of HMS0014 cells in PuraMatrix in vitro

1) ALP activity increased with elapsed time. A significant difference was evident at 14 days experiment $(* * \mathrm{p}<0.01)$.

2) Time-dependent increase of $\mathrm{Ca}$ volume was evident. $\mathrm{A}$ significant difference of $\mathrm{Ca}$ volume was observed in the $14^{\text {th }}$ day of experiment $(* \mathrm{p}<0.05)$.

3) The OC volume abruptly and significantly increased at 14 days in the experimental group $(* * \mathrm{p}<0.01)$.

Fig. 1. Time-dependent changes of ALP activity, Ca volume and Osteocalcin (OC) volume in monolayer cultures (Fig. 1a) and 3-D (in PuraMatrix) cultures (Fig. 1b) of HMS0014 cells in control and experimental (inducing-conditioned) groups. The results indicated that mineralization of the ECM prominently progressed between day 7 and day 14, and acquired more $\mathrm{Ca}$ and OC volumes in the 3-D cultures.

group was demonstrated in the day 14 of the ECM secreted by cells cultured in 3-D PuraMatrix scaffold $(* * p<0.01)$. By comparison of the obtained data, the results indicated that the $\mathrm{OC}$ volume was $4 \times$ higher in 3 -D cultures than in monolayer cultures (Figs. 1A \& 1B).

The results indicated that the $3 \mathrm{D}$-cultured proliferating HMS0014 cells was actively induced to differentiate into Os-like cells, which abundantly secreted mineralizing ECM between day 7 and day 14 of culture (Fig. 1b). Collectively, the results indicated that mineralization of the ECM prominently progressed between day 7 and day 14 of experiment, and the $\mathrm{Ca}$ volume and $\mathrm{OC}$ volume in the ECM of cells cultured with 3-D PuraMatrix scaffold were higher than in that observed in monolayer cultures with data of $2 \times$ and $4 \times$, respectively (Figs. 1 A \& 1B).

\section{Histological findings of the inducing-conditioned HMS0014 cells}

1. Phase contrast microscopy of proliferation and mineralization of the cells in induction condition in vitro (Fig. 2) 1) Monolayer cultures induced the HMSO014 cells to actively proliferate and differentiate into polygonal cells secreting abundant ECM. On the other hand by using Alizarin red (AR) vital staining (AR-S) of the secreted ECM, the phase contrast microscopy demonstrated that the occurrence of mineralization loci had been initiated in the $1^{\text {st }}$ day and became markedly deposited in the $7^{\text {th }}$ day (Fig. 2A).

2) 3-D culture of HMS0014 cells with PuraMatrix induced extension of cell processes, gradual aggregation and sedimentation of calcification loci in the ECM since the 3 days culture (Fig. 2B).

2. Scanning electron microscopy (SEM) of culturing HMS0014 cells on the modified surface of Ti-alloy and Ti discs (i.e., Disc-AO, Disc-HA, Disc-SPI; Fig. 3)

1) At 60 minutes culture, the inducing-conditioned cells migrated towards depressions of the disc surface, loosely proliferated and differentiated to spherical (e.g., cells on Disc-HA and Disc-SPI surfaces; $10 \mu \mathrm{m}$ in diameter, approx.) and flat, polygonal (e.g., cells on Disc-AO surface; $40 \mu \mathrm{m}$ in diameter, approx.) cells with a bulging cell body sending out many minute filopodia (e.g., Disc-SPI) and some lamellipodia (e.g., Disc-AO, Disc-HA) to attach with the substrate in the concavities (Fig. 3A). 
Proliferation and differentiation (monolayer culture)
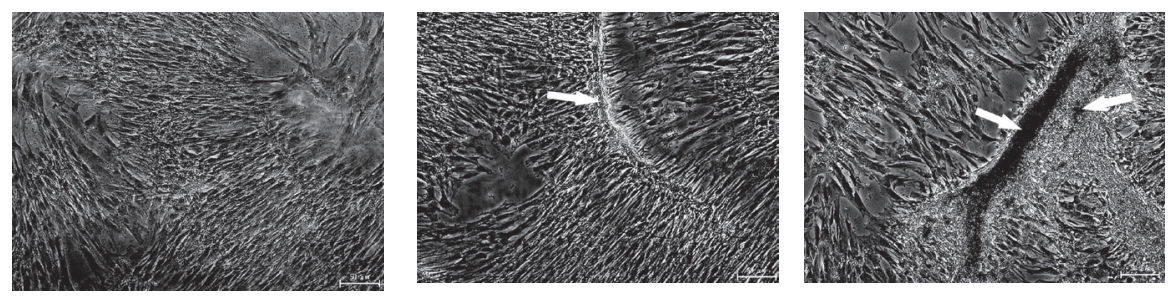

Scale bar: $50 \mu \mathrm{m}$

Mineralization (monolayer culture, AR-S vital staining)

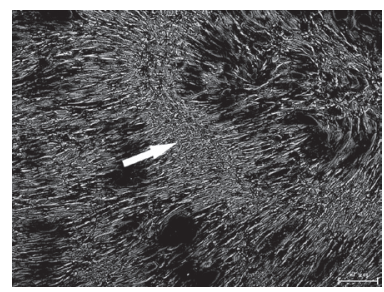

Day 1

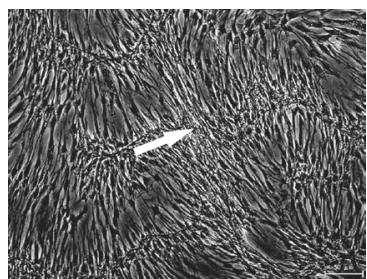

Day 3

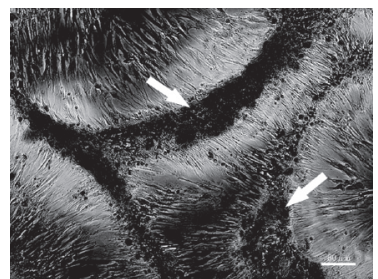

Day 7
Scale bar: $50 \mu \mathrm{m}$

Fig. 2A. PCM studies on proliferation and differentiation, and mineralization of HMS0014 cells (under inducing condition, monolayer culture; arrow: calcification locus; scale bar: $50 \mu \mathrm{m}$ )

The PCM observed that polygonal HMS0014 cells proliferated actively, and were differentiated into cells secreting abundant extracellular matrix (ECM) in induction condition. Alizarin red S (AR-S) staining indicated that mineralization initiated on day 1 and became markedly deposited in the ECM on day 7 experiment.

3-D cultures of HMS0014 cells in PuraMatrix scaffold

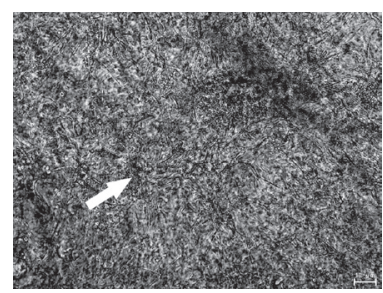

Day 1

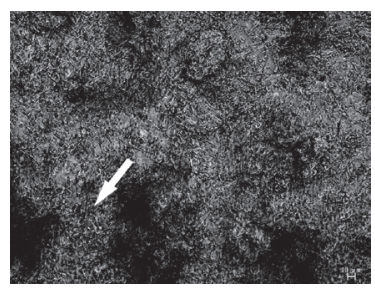

Day 3

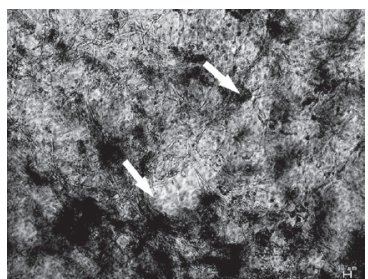

Scale bar: $10 \mu \mathrm{m}$

Fig. 2B. PCM of HMS0014 cells cultured with PuraMatrix (under inducing condition, 3-D culture; arrows: calcification loci; scale bar: $10 \mu \mathrm{m}$ ) The PCM showed that cells extended the cell processes three dimensionally. Gradual aggregation and sedimentation of calcification loci (arrows) were evidently observed in the ECM.

Fig. 2. Phase contrast microscopy (PCM) of time-dependent changes of HMS0014 cells and the ECM in monolayer (Fig. 2a) and 3-D PuraMatrix scaffold (Fig. 2b) cultures.

2) At 180 minutes culture, the HMS0014 cells actively proliferated and differentiated into large flat and polygonal shapes $(30 \mu \mathrm{m} \times 90 \mu \mathrm{m}$ to $100 \mu \mathrm{m} \times 200 \mu \mathrm{m}$, approx.), so that spread the Disc-AO, Disc-HA and Disc-SPI surfaces with prominent lamellipodia and many fine dendritic filopodia to employ cell-to-substrate and intercellular attachments (Fig. 3B).
3. Fluorescent immunohistology of culturing HMS0014 cells on the modified surface of Ti-alloy and Ti discs (i.e., Disc-AO, Disc-HA, Disc-SPI; Fig. 4)

CD51 ( $\alpha_{v}$ integrin) and F-actin immunoreactivities of cells with massive extension to attach on the Disc-AO, Disc-HA and Disc-SPI surfaces at 60 and 180 minutes under inducing condition were evident; the co-expression 

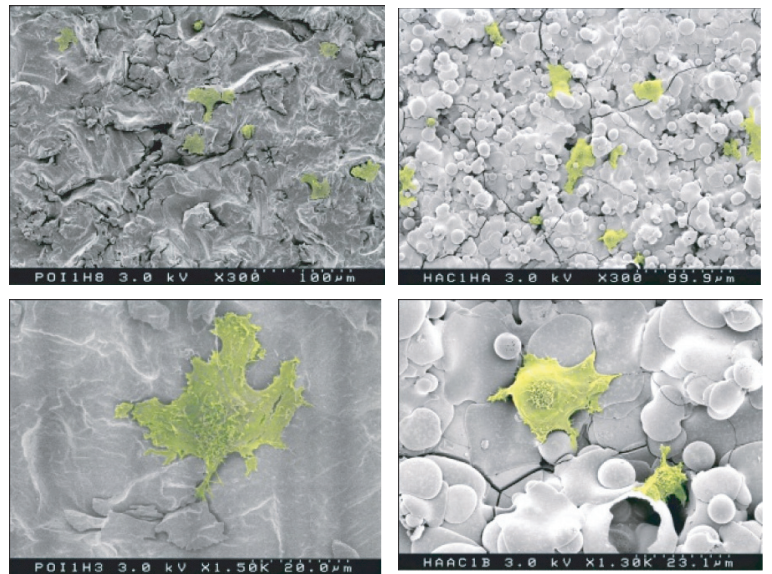

60-min Disc-AO

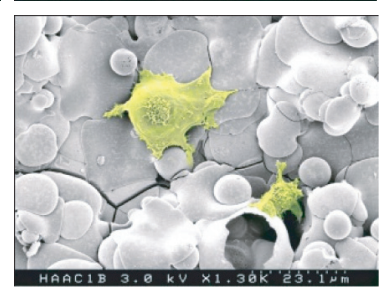

60-min Disc-HA
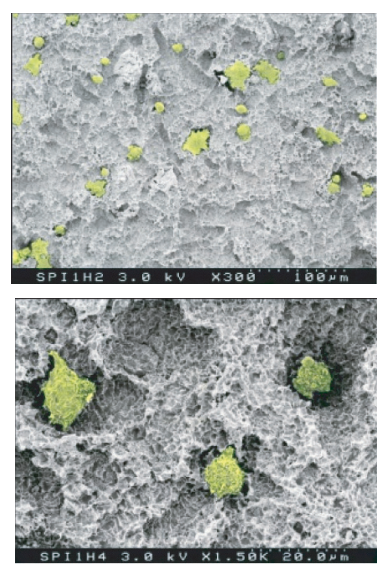

60-min Disc-SPI
Fig. 3A. SEM showing HMS0014 cells were induced to attach with the substratum of Ti discs after 60 minutes of culture using GBR method.

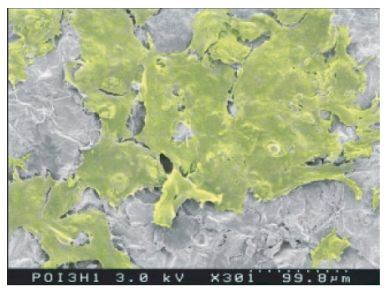

180-min Disc-AO

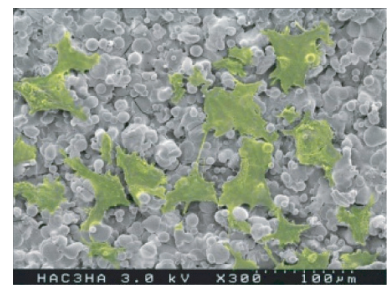

180-min Disc-HA

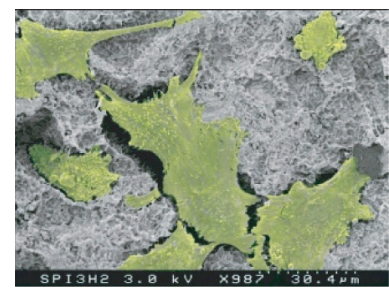

180-min Disc-SPI
Fig. 3B. SEM showing HMS0014 cells were induced to attach with the substratum of $\mathrm{Ti}$ discs after 180 minutes culture using GBR method.

Fig. 3. Scanning electron microscopy (SEM) showing time-dependent (Fig. 3a: 60-min specimens, Fig. 3b: 180-min specimens) morphological changes of HMS0014 cells, which were induced to proliferate and differentiate into mature Ob-like cells on the substratum of titanium discs (i.e., Disc-AO, Disc-HA and Disc-SPI). The loosely distributed spherical-to-polygonal HMS0014 cells (10 to $40 \mu \mathrm{m}$ in diameter, avg.; 60-min specimens) subsequently differentiated into flat, large polygonal cells with prominent lamellipodia and dendritic filopodia $\left(30 \times 90 \mu \mathrm{m}^{2}\right.$ to $100 \times 200 \mu \mathrm{m}^{2}$, approx.; 180-min specimens).

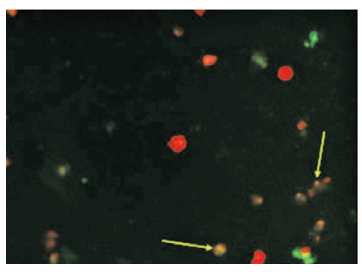

60-min Disc-AO

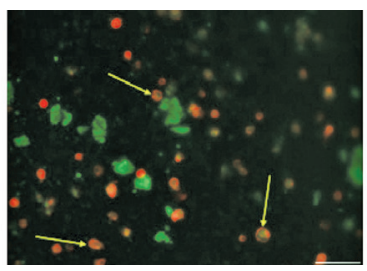

60-min Disc-HA

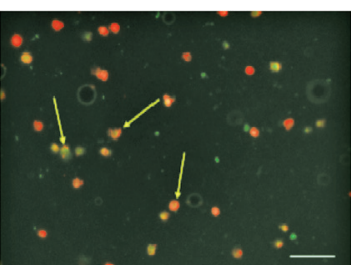

60-min Disc-SPI
Scale bar: $70 \mu \mathrm{m}$

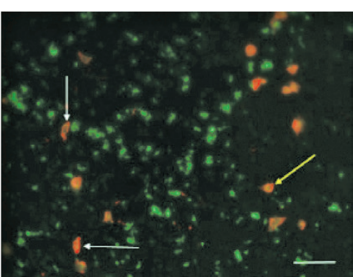

180-min Disc-SPI
Scale bar: $70 \mu \mathrm{m}$

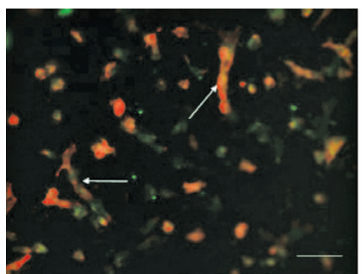

180-min Disc-AO

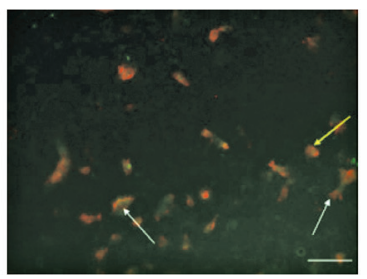

180-min Disc-HA

Fig. 4. Fluorescent immunohistology demonstrating the expression of CD51 ( $\alpha$ v integrin; green) and F-actin (red) immunoreactivities of the 60-min and 180-min specimens (i.e., Disc-AO, -HA and -SPI specimens (yellow arrow: CD51/F-actin co-expression, white arrow: polygonal cell with massive extension; scale bar: $70 \mu \mathrm{m})$. 
of CD51 and F-actin co-expression was in particularly demonstrated in the 180-min specimens (Fig. 4).

\section{Discussion}

Many studies on the endosseous dental implantation have demonstrated that pure Ti and Ti-alloy IP surface modified with bioactive substrates acquired an immediately mechanical stability to enhance cell attachment, improve the host-to-IP response and thereby optimize osseointegration $^{2,5,13,26-30)}$. Additionally, many histological studies indicated that osseointegration begins with recruitment and migration of osteogenic lineage cells, which adhered and spread, proliferated, and subsequently differentiated into osteoblast $(\mathrm{Ob})$-like cells which initiated osteogenesis by cell and tissue ingrowth onto the IP surface; the mature Ob-like cells changed in shape concomitant with differentiation and expressed osteocalcin gene in the mineralizing phase $\mathrm{e}^{1-3,30-33)}$. Meanwhile, interfacing specific receptor proteins (e.g., integrins) and cytoskeleton proteins (e.g., actin filaments) have been elucidated to be proteins closely related with the development of focal contacts, which play important roles in signal transduction and thereby induce adhesion, spreading, cell migration and consequently regulate cell growth and differentiation of the Ob-like cells attached on the IP substratum $^{32-34)}$.

In our recent studies, we have 3-D cultured rat JCRB119:KUSA/A1 cells with a collagen gel scaffold (i.e., Cellmatrix Type I-A) on dental IPs with different substrates (i.e., IP-AO, IP-HA, IP-SPI) to create a microenvironment conducting contact and distant osteogenesis since the first three days of culture by using the GBR tissue-engineering methods. Consequently, we observed that the GBR methods engineered new bone formation without intervening soft tissue in the peri-IP osteogenesis, owing to that there had a non-collagenous matrix been essentially precipitated to secure the substratum layer of IPs for contact osseointegration. Hence, we hypothesized that the GBR peri-IP osteoid tissue might be employed to an en-bloc biocompatible and biodegradable tissue, which could consequently be remolded and modeled to benefit optimal osseointegration of the dental IP therapy ${ }^{19,20)}$. By using the similar GBR tissue-engineering methods, in this study we performed monolayer culture of human osteogeneic HMS0014 cells on Ti-alloy and Ti discs (i.e., Disc-AO, Disc-HA, Disc-SPI), and the present SEM demonstrated that the HMS0014 cells grew and became differentiated into flat, polygonal cells having long filopodia (cytoplasm supported with long F-actin bundles) and defined wide-spreading lamellipodia (extended cytoplasm supported with a F-actin meshwork) to facilitate cell motility on the disc surface ${ }^{34,35)}$. On the other hand, the present fluorescent immunohistology of the expression of $\alpha_{\mathrm{v}}$ integrin and $\mathrm{F}$-actin indicated that we engineered actin- rich HMS0014 cells on the Ti/Ti-alloy discs with different modifications (i.e., Disc-AO, Disc-HA and Disc-SPI). The proliferating cells actively migrated and dispersed along the bioactive interface with participation to bind, initiate and establish focal adhesions with the modified substratum within 180 minutes of culture ${ }^{30,33,35-37)}$.

Furthermore, we confirmed that the ECM of the osteogenic linage HMS0014 cells in experimental (under inducing condition) monolayer cultures showed an increase of osteocalcin activity through the experimental term, and the cells were actively proliferated differentiating into Obs with an abrupt and significant increase of ALP activity and $\mathrm{Ca}$ volume in particular at 14 days experiment. Similar results were also obtained in studying conditioninduced 3-D cultures of the HMS0014 cells in hydrogel PuraMatrix (3-D Matrix Japan), which has been elucidated to be a peptide-based scaffold enhancing cellbiomaterial interactions to foster growth and differentiation of cultured mesenchymal cells ${ }^{21-25}$. On the other hand, in accordance with the assay of the osteoinductive property of the experimental HMS0014 cells, the present phase contrast microscopy of both the monolayer and 3-D cultures also showed that polygonal HMS0014 cells had actively proliferated and been differentiated to secrete abundant ECM with sedimentation and aggregation of calcification loci since day 1 of culture; subsequently, the ECM became markedly mineralised since day 7 of experiment. Collectively, these results indicated that HMS0014 cells were condition-induced to differentiate into mature Ob-like cells to secrete ECM which was prominently mineralized between day 7 and day 14 in both monolayer and 3-D cultures. Furthermore, we estimated that experimental HMS0014 cells cultured with PuraMatrix produced more mineralizing ECM on day 14.

We confirmed that the present GBR engineered HMS0014 cells to obtain extensive cell attachment on different microtextured Ti discs within 180 minutes, thereby we surmised that the Obs might be further regulated to initiate peri-Ti osteogenesis at the disc surfaces since day 1 of culture. Further in vitro and in vivo studies to clarify peri-IP osseogenesis, bone modeling and remodeling following osseointegration of the IP-tissue interfaces should be the next steps in programming of GBR tissues for the dental implant therapy.

\section{Acknowledgements}

The laboratory animal, morphological research, tissue culture facilities and dental bioscience facility I of the Institute of Dental Research, Osaka Dental University, were used to perform this study. The authors gratefully acknowledge Mr. Hideaki Hori (Research Technologist, the Institute of Dental Research, Osaka Dental University). 


\section{References}

1) Davies JE: In vitro modeling of the bone/implant interface. Anat Rec 1996; 245:426-445.

2) Nasatzky E, Gultchin J, Schwartz Z: The role of surface roughness in promoting osteointegration. Refuat Hapeh Vehashinayim 2003; 20:8-19, 98.

3) Davies JE: Understanding peri-implant endosseous healing. J Dent Educ 2003; 67:932-949.

4) Albrektsson T, Berglundh T, Lindhe J, Wennerberg A; Osseointegration: Histric Background and Current Concepts, Surface Topography of Titanium Implants. In: Lindhe J, Karring T, Lang P, eds, Clinical Periodontology and Implant Dentistry. 4th ed. Oxford, UK, Blackwell Munksgaard, a Blackwell Publishing Company 2003; 809-828

5) Marco F, Milena F, Gianluca G, Vittoria O: Peri-implant osteogenesis in health and osteoporosis. Micron 2005; 36:630-644.

6) Ferguson SJ, Langhoff JD, Voelter K, von Rechenberg B, Scharnweber D, Bierbaum S, Schnabelrauch M, Kautz AR, Frauchinger VM, Mueller TL, von Lenthe H, Schlottig F: Biomechanical comparison of different surface modifications for dental implants. Int $\mathrm{J}$ Oral Maxillofac Implants 2008; 23:1037-1046.

7) Langhoff JD, Voelter K, Scharnweber D, Schnaberlrauch M, Schlottig F, Hefti T, Kalchofner K, Nuss K, von Rechenberg B: Comparison of chemically and pharmaceutically modified titanium and zirconia implant surfaces in dentistry: a study in sheep. Int $\mathrm{J}$ Oral Maxillofac Surg 2008; 37:1125-1132.

8) Koyano K, Matsushita Y, Ayukawa Y; Basic science for the dental implantology. In: Koyano K, Matsuura M, eds, Essential oral implantology Tokyo, Japan, Ishiyaku Publishers, Inc. 2009; 10-17 (in Japanese).

9) Murai K, Takeshita F, Ayukawa Y, Kiyoshima T, Suetsugu T, Tanaka T: Light and electron microscopic studies of bone-titanium interface in the tibiae of young and mature rats. J Biomedical Mater Res 1996; 30:523-533.

10) Ferretti M, Palumbo C, Contri M, Marotti G. Static and dynamic osteogenesis: Two different types of bone formation. Anat Embryol 2002; 21-29.

11) Inoue $\mathrm{T}$; Mechanisms of metabolism and wound healing. In: Yamazaki M, Takahashi T, Katsuyama H, Inoue T, Hayashi Y, eds, The Ultimate Guide to Implant Dentistry Tokyo, Japan, Ishiyaku Publishers Inc., 2004; 38-53 (in Japanese).

12) Inoue $\mathrm{T}$; Histology (Bone Soft tissue). In: Akagawa $Y$ et al., eds, Fundamental concepts and techniques of oral implants Tokyo, Japan, Ishiyaku Publishers, Inc., 2005; 47-59 (in Japanese).

13) Joos U, Meyer U: New paradigm in implant osseointegration. Head Face Med 2006; 2:19.

14) Tsutsumi S, Shimazu A, Miyazaki K, Pan H, Koike C, Yoshida E, Takagishi K, Kato Y: Retention of multilineage differentiation potential of mesenchymal cells during proliferation in response to FGF. Biochem Biophysic Res Communications 2001; 288:413-419.

15) Kim GS, Kumabe S, Iwai Y. An experimental study on transplantation of human dental pulp-derived cells using alginate scaffold. J Jpn Assoc Regenerative Dent 2005; 3:41-56 (in Japanese).

16) Fujiwara $S$, Kumabe $S$, Iwai $Y$ : Isolated rat dental pulp cell culture and transplantation with an alginate scaffold. Okajimas Folia Anat Jpn 2006; 83:15-24.

17) Aikawa F, Iwai Y: Capsaicin activates transient receptor potential vanilloid subtype 1 (TRPV1) of rat bone marrow-derived stromal cells (BMSCs) in vitro. J Oral Tissue Engin 2008; 6:41-55.

18) Nakatsuka M, Mikami T, Hashimoto Y, Kon-I H, Chen HS, Hsiao SY, Kumabe S, Huang ST, Iwai Y, Huang HC: A preliminary study of osseointegration in the dental implant therapy in vivo - culture of mouse KUSA/A1 cells on titanium plates with different surface modifications -. TWJ Oral Med Sci 2009; 25:4-19.

19) Nakatsuka M, Hashimoto $Y$, Kumabe S, An C, Ueda K, Mikami H, Huang HC, Iwai Y: The Study of Cell Attachment and Hard Tissue Formation on the Dental Implant Surface [Abstract]. Kaibougaku Zasshi 2010; 85(Suppl): 177 (in Japanese).

20) Mikami T, Kumabe S, Iwai Y: A study of osseointegration - 3-D of KUSA/A1 cells with a collagen scaffold on titanium implants with different surface modifications -. J Oral Tissue Engin 2010; 8:60-73.

21) Kisiday J, Jin M, Kurz B, Hung H, Semino C, Zhang S, Grodzinsky AJ: Self-assembling peptide hydrogel fosters chondrocyte extracellular matrix production and cell division: implications for cartilage tissue repair. Proc Natl Acad Sci USA 2002; 99:9996-10001.

22) Holmes TC: Novel peptide-based biomaterial scaffolds for tissue engineering. Trend Biotechnol 2002; 20:16-21.

23) Zhang S: Fabrication of novel biomaterials through molecular selfassembly. Nat Biotechnol 2003; 21:1171-1178.

24) Kisiday JD, Jin M, DiMicco MA, Kurz B, Grodzinsky AJ: Effects of dynamic compressive loading on chondrocyte biosynthesis in self-assembling petide scaffolds. J Biomech 2004; 37:595-604.

25) Bokhari MA, Akay G, Zhang S, Birch MA: The enhancement of osteoblast growth and differentiation in vitro on a peptide hydrogel-polyHIPE polymer hybrid material. Biomaterials 2005; 26:5198-5208.

26) Ong JL, Villarreal DR, Cavin R, Ma K: Osteoblast responses to as-deposited and heat treated sputtered $\mathrm{CaP}$ surfaces. J Mater Sci Mater Med 2001; 12:491-495.

27) Fillies T, Wiesmann HP, Sommer D, Joos U, Meyer U: Osteoblast reaction on SLA and microgroved implant surfaces. Mund Kiefer Gesichtschir 2005; 9:24-28.

28) Graf HL, Stoeva S, Armbruster FP, Neuhaus J, Hilbig H: Effect of bone sialoprotein and collagen coating on cell attachment to TICER and pure titanium implant surfaces. Int J Oral Maxillofac Surg 2008; 37:634-640.

29) Coelho PG, Granjeiro JM, Romanos GE, Suzuki M, Silva NR, Cardaropoli G, Thompson VP, Lemons JE: Basic research methods and current trends of dental implant surface. J Biomed Mater res B Appl Biomater 2009; 88:579-596.

30) Issac J, Loty S, Hamdan A, Kokubo T, Kim HM, Berdal A, Sautier JM: Bone-like tissue formation on a biomimetic titanium surface in an explant model of osteoconduction. J Biomed Mater Res A 2009; 89:585-593.

31) Jayaraman M, Meyer U, Bühner M, Joos U, Wiesmann HP: Influence of titanium surfaces on attachment of osteoblast-like cells in vitro. Biomaterials 2004; 25:625-631.

32) Meyer U, Büchter A, Wiesmann HP, Joos U, Jones DB. Basic reactions of osteoblasts on structured material surfaces. Eur Cell Mater 2005; 9:39-49.

33) Salido M, Vilches Jl, Gutiérrez JL, Vilches J: Actin cytoskeletal organization in human osteoblasts grown on different dental titanium implant surfaces. Histol Histopathol 2007; 22:1355-1364.

34) Anselme K: Osteoblast adhesion on biomaterials. Biomaterials 2000; 21:667-681

35) Zhu XL, Eibl O, Scheideler L, Geis-Gestorfer J: Charactrization of nano hydroxyapatite/collagen surfaces and cellullar behaviors. J Biomed Mater Res A 2006; 79:114-127.

36) Boyan BD, Lossdörfer S, Wang L, Zhoa G, Lohmann CH, Cochran DL, Schwartz Z: Osteoblasts generate an osteogenic microenvironment when growth on surfaces with rough microtopographies. Eur Cell Mater 2003; 6:22-27.

37) Diener A, Nebe B, Lüthen F, Becker P, Beck U, Neumann HG, Rychly J: Control of focal adhesion dynamics by material surface characteristics. Biomaterials 2005; 26:383-392. 